\title{
The role of familiarity in determining typicality
}

\author{
BARBARA C. MALT and EDWARD E. SMITH \\ Stanford University, Stanford, California 94305
}

\begin{abstract}
Ashcraft (1978b) found that people tend to know more properties of instances they rate as typical of a category than of instances they rate as atypical. This suggests that variations in typicality result from variations in familiarity. Three experiments are presented that challenge or qualify this suggestion. Experiment 1 showed that subjects sometimes produce more properties for items they rate as low in typicality. Experiment 2 showed that in a large, random sample of items, there was a tendency to produce fewer properties for atypical items, but Experiment 3 indicated that part of the reason for this result was a response bias to assign low typicality ratings to unfamiliar words, rather than a reflection of low perceived typicality of the referents themselves. These results suggest that variations in typicality can exist independent of variations in familiarity, although familiarity may also play a role.
\end{abstract}

All members of a semantic category are not equally representative or typical of that category: A peach is a more typical fruit than a pomegranate, and a robin is a more typical bird than a roadrunner. By now it is well established that subjects show strong agreement in their ratings of how typical members of semantic categories are. It is also well established that rated typicality predicts performance in a wide variety of tasks, such as reaction time to verify category membership, order of learning by children, and order and probability of output in free listing to category names (Mervis \& Rosch, 1981; Rips, Shoben, \& Smith, 1973; Rosch, 1975; Rosch \& Mervis, 1975). Understanding what determines typicality is thus a step toward understanding the principles by which information in semantic memory is acquired and organized.

Most explanations of typicality have focused on the property structure of categories, examining the properties of the category members or those of the category itself (e.g., Rosch \& Mervis, 1975; Smith, Shoben, \& Rips, 1974). Rosch and Mervis, for example, suggested that typicality is based on the distribution of properties among category members. Typical members have properties that are common to many category members, and atypical members have properties that are common to few category members. In support of this proposal, they found a strong correlation between typicality ratings and family resemblance scores, a measure of overlap of an exemplar's properties with the properties of other category members.

An alternative to a structural account, however, is a

This research was supported, in part, by U.S. Public Health Service Grant MH-19705. Requests for reprints should be sent to Barbara C. Malt, Department of Psychology, Stanford University, Stanford, California 94305. We thank Kathy Hemenway, Larry Barsalou, and Greg Murphy for helpful comments on an earlier draft of this paper. E. E. Smith is now at Bolt Beranek and Newman Inc., Cambridge, Massachusetts. familiarity-based explanation of typicality. Most college students encounter peaches more often than pomegranates and robins more often than roadrunners. Those category members that are most frequently seen, talked about, or interacted with may be those judged most typical.

Until recently, this kind of explanation has been discounted, largely on the basis of artificial category experiments and word frequency data. Mervis, Catlin, and Rosch (1976), for example, found no correlation between Kurera and Francis (1967) word frequency and rated typicality for members of common categories. Rosch, Simpson, and Miller (1976) demonstrated that when frequency of presentation was manipulated for members of artificial categories, typicality ratings reflected structural variables rather than frequency. None of these results, however, directly addressed the problem of what determines typicality for real-world categories. Frequency counts of the occurrence of words in prose need not necessarily reflect how familiar or common in the environment an object is; we may tend, for instance, to write disproportionately about the uncommon and unusual. It also is not clear to what extent the artificial category results are indicative of the real-world phenomenon, since there may be a much greater range in familiarity for members of real-world categories than was used in the artificial category research.

Recent work has revived familiarity explanations of typicality. McCloskey (1980) asked subjects to rate the familiarity of meaning of words used in several published semantic memory experiments. He found a wide range of familiarity, although word frequency and length had been carefully controlled in these experiments. This familiarity measure seemed to correlate with rated typicality, and the effect of typicality on reaction time was reduced substantially (but not eliminated) when familiarity was partialled out.

It is not clear in McCloskey's (1980) study whether 
subjects were rating how familiar the word was or how familiar the real-world referent was. Ashcraft (1978b) investigated familiarity of referents of words more directly. He had subjects free list properties for highand low-typical members of 17 semantic categories and found that category members rated low in typicality had fewer properties listed than did members high in typicality. Furthermore, the mean number of properties generated was more highly correlated with typicality ratings than were other measures, including property overlap with the superordinate. It was also a better predictor of reaction time in a property verification task than rated typicality was (Ashcraft, 1978a). Ashcraft concluded that the larger number of properties listed for typical than for atypical members reflects the fact that subjects are more familiar with the typical members and hence know and can produce more information about them than they can about the less typical category members. He further suggested that the amount of readily accessible information for an exemplar, reflected in the mean number of properties produced, is the variable underlying standard typicality effects in the semantic memory literature.

Ashcraft's (1978a, 1978b) data provide the best available support for a familiarity account of typicality ratings. If familiarity can explain typicality ratings, then the pattern of distribution of properties discussed by Rosch and Mervis (1975) and others may be artifactual. Those birds, trees, vehicles, and so on, that are familiar (and therefore typical) are most likely familiar because they are frequent, and frequent because they have properties that make them particularly useful or well suited to the environment. The properties that make one member of a category well suited to a given environment will tend to be the same properties that make another member well suited, and hence the most frequent or familiar members will have many properties in common. The less well suited members, on the other hand, may have many different properties that lead to their lesser suitability. Rosch and Mervis' (1975) family resemblance distribution of properties is thus obtained. Greater familiarity with certain items can also easily account for typicality effects on verification times and order of learning. Verification times may be speeded by many well integrated pathways in the representation of the concept, as Ashcraft (1978a) suggests, and children are more likely to be taught, and to have more practice with, the names of common, familiar objects.

Even if familiarity is an important determinant of typicality, can it account for all the variance in typicality ratings? Many of the category members rated as atypical in the Rosch (1975) norms do appear to be less familiar than the typical ones. However, certain items, such as chickens, pumpkins, and coconuts, that are rated low in typicality (for the categories birds, vegetables, and fruit, respectively) appear to be as frequent and familiar as many of the more typical category members. Glass and Meany (1978) found that atypical exemplars seem to be of two types: high-imagery, apparently familiar items, and low-imagery, less familiar items. These instances suggest that some factor other than familiarity is accounting for a certain portion of the variance in typicality ratings.

The experiments to be presented used Ashcraft's (1978b) procedure of free listing properties to category members to examine these issues and investigate the generality of Ashcraft's results. Ashcraft (1978b) used only three typical and three atypical members of each category and did not specify his sampling procedure. It is not clear to what extent his data reflect the pattern of familiarity across a wide range of typicality values within a category. Our first study sought to determine whether there is necessarily a positive correlation between typicality and number of properties produced for members of categories. The second study tested whether such a correlation holds for large, random samples of category members. The third study examined a possible confounding between familiarity of the word and familiarity of the word's referent.

\section{EXPERIMENT 1}

The purpose of this experiment was to determine whether familiarity is necessarily correlated with typicality ratings for large samples of category members that span the typicality range. Two categories were used for which exemplars had been chosen to be at least somewhat familiar to college student subjects. Rated typicality was then correlated with the mean number of properties produced to each category member. If the main determinant of typicality is an item's familiarity, then rated typicality and mean number of properties produced should correlate positively even when the sample has been chosen such that no exemplars are completely unknown to subjects.

\section{Method}

Subjects. Twenty Stanford students generated properties for category members, and an additional 19 students rated the category members for typicality.

Materials. The items were 20 members of the category "furniture" and 15 members of the category "bird." The 20 furniture members were the sample used by Rosch and Mervis (1975), which had been selected to span the typicality range. The bird members were chosen from Rosch's (1975) norms to span the typicality range and yet not be unknown to college students.

Procedure. Subjects providing typicality ratings were given sheets of paper with the category name typed at the top and the members listed in random order below. They were asked to rate each member for how typical an example of the category it was. Two random orders of members were used for each category. Ratings were made on a scale of 1 to 7 , with 7 indicating highest typicality.

Property lists were collected from a separate group of sub jects. Each item was typed at the top of a sheet of paper. Subjects were given $75 \mathrm{sec}$ to list all the properties they could think of for each item. Each subject was given all 35 category members (20 furniture and 15 bird members randomly intermixed) in a different random order. 


\section{Results and Discussion}

The total number of properties listed for each item was averaged over subjects to yield a mean for that item. Pearson correlations were then calculated between the mean number of properties produced and mean typicality ratings for each item. (See Appendix A for a list of the items, typicality ratings, and mean number of properties produced.) For the bird category, the correlation between properties produced and typicality was $-.63(\mathrm{p}<.025)$; for furniture, the correlation was -.23 ( $p>.10$ ). Contrary to Ashcraft's (1978b) results, then, the number of properties increased as typicality decreased for both of these categories. These results are not due to the presence of items at the low end of the typicality scale that are actually typical members of some other category: Eliminating "bat" from bird and the electrical appliances (stove, telephone, clock, and radio) from furniture did not substantially change the two correlations $(\mathrm{r}=-.61$ and $\mathrm{r}=.25 ; \mathrm{p}<.025$ and $\mathrm{p}>.10)$.

Thus, atypical members of a category are not necessarily those that people have had little contact with and know little about. Our atypical furniture members included the items "lamp," "piano," and "stove," which are likely to be no less frequent in the environ. ment than sofas or desks, and for which equally as many properties were listed. In the bird sample, subjects produced surprisingly few properties for some of the birds they rated as typical, such as swallows and bluebirds, which suggests that these items were actually relatively little known to subjects, except that they had generally ordinary bird-like properties. Subjects knew much more about some of the birds that were low in typicality, such as chickens and penguins. ${ }^{1}$ Indeed, the contrast between detailed knowledge of salient, distinctive properties for atypical members and only general knowledge for some typical members was great enough to produce the negative correlations.

What factors account for the discrepancy between these results and those of Ashcraft (1978b)? One likely possibility is sampling procedure. The category members used in Experiment 1 were not a random sampling of all possible members of the two categories; rather, we chose the bird items so that they would be recognizable to college students, and it is likely that the furniture items had been selected similarly by Rosch and Mervis (1975). It may be that, while familiarity alone does not determine typicality, it does play some role. In a completely random sampling of items that range widely in familiarity, those items about which subjects know the least may tend to be at the lower end of rated typicality.

\section{EXPERIMENT 2}

Experiment 2 tested whether, for a random sample of 15 items from each of eight categories, a positive correlation between typicality ratings and number of properties listed would be found.

\section{Method}

Subjects. The subjects were 260 Stanford undergraduates who participated either for course credit or as paid volunteers. Two hundred forty of them provided property lists, and 20 rated the stimulus items for typicality.

Materials. Eight categories were chosen from among the 17 used by Ashcraft (1978b). Four of them (furniture, vehicle, fruit, and clothing) were chosen to be at superordinate level of abstraction, and four (tree, bird, fish, and flower) were at the basic level (Rosch, Mervis, Gray, Johnson, \& Boyes-Braem, 1976). ${ }^{2}$ For each category, 15 members were chosen. Six of them were the six used by Ashcraft (1978b). An additional nine were randomly sampled from Battig and Montague (1969), primarily using exemplars with a production frequency greater than or equal to nine.

The words were typed at the top of sheets of paper that were assembled into packets. Each packet contained one member from each of the eight categories. Packets of items were constructed such that no more than 4 of the 240 packets contained the same set of items and no more than 2 of those 4 contained the items in the same order.

Procedure. Typicality ratings were collected in the same manner as in Experiment 1. Each subject rated all eight categories in a different random order. There were two random orderings of each set of 15 category members. Half the subjects received one set of random orderings, and half received the other.

Subjects providing property lists were each given a packet containing one member from each of the eight categories, as described above. They were given $75 \mathrm{sec}$ to list properties for each member. They were explicitly told to write as much as they knew for any item they were uncertain about.

\section{Results and Discussion}

Mean typicality ratings and mean number of properties produced were calculated for each item, and the correlation between these two measures was obtained for each category. The items, ratings, and mean number of properties produced are given in Appendix B. In contrast to Experiment 1 , the correlation coefficients were positive for all eight categories, indicating that subjects knew more about exemplars high in typicality than about those low in typicality. For five of the categories, the correlations were significant: bird, $r=.68$; flower, $r=.69$; fruit, $r=.66$; tree, $r=.67$; and vehicle, $r=.62(p<.025$ in all cases). The remaining three correlations were positive but nonsignificant: clothing, $r=.24$; fish, $r=.07$; and furniture, $r=.36$ ( $>.10$ in all cases). There was no systematic difference between basic- and superordinate-level categories. Also, as expected, the degree of familiarity varied much more for these items than for the set in Experiment 1. Whereas the mean number of properties listed ranged from 5.95 to 9.60 in Experiment 1, the range for Experiment 2 was 1.56 to 11.63 . Thus, when the items range from very familiar to very unfamiliar, typicality ratings do show substantial correlation with familiarity, as Ashcraft (1978b) found.

The change in sampling procedure from Experiment 1 to Experiment 2 reversed the direction of the critical correlations. It is not clear, however, exactly how the greater range of familiarity in Experiment 2 stimuli might have changed the direction of the results. The familiarity explanation of typicality assumes that 
subjects have at least a rough idea of the appearance of the referent of the item they are rating as low in typicality; it is the relative infrequency of that referent in the environment that leads it to be perceived as less typical. Ability to list fewer properties is a by-product and indicator of this lesser familiarity. However, the extreme lack of knowledge evidenced by many subjects for a number of Experiment 2 stimuli suggests an alternative explanation. If subjects have no idea what the referent of a word is, they cannot make a typicality judgment about the referent itself and perhaps resort to a strategy of assigning low ratings to such words. Experiment 3 was therefore designed to distinguish between the two possibilities. ${ }^{3}$

\section{EXPERIMENT 3}

In most typicality rating tasks, subjects are given a scale that forces them to place every item somewhere on the typicality continuum. Subjects are never given a chance to indicate items with which they are completely unfamiliar. Experiment 3 collected a new set of typicality ratings in which an alternative response to a typicality number was " $U$," indicating that the rater was too unfamiliar with the referent of the word to rate it on the scale. If the referents of some low-rated items in Experiment 2 were so unfamiliar that subjects had no idea of their appearance, then these items should receive "U" ratings from the present group of subjects. Furthermore, when those items rated as " $U$ " by a substantial number of subjects are removed from the calculations, the correlations between familiarity and typicality should be reduced. On the other hand, if the typicality ratings in Experiment 2 truly reflect perceived typicality of the referent, then the number of "U" ratings should be minimal and there should be no tendency for them to cluster at the lower end of the typicality range.

\section{Method \\ Subjects. Twenty Stanford undergraduates participated in partial fulfillment of a course requirement. \\ Materials. Packets of eight rating sheets, identical to those in Experiment 2, were used. \\ Procedure. Subjects were given standard typicality rating instructions, but were also told, "For any item which is unfamil- iar enough to you that you don't feel able to accurately rank it with respect to the others, place a $U$ (for 'Unfamiliar') in the blank instead of a number."}

\section{Results and Discussion}

The number of times each item was given a $U$ rating was tabulated. Of 120 items, 30 were rated as $\mathrm{U}$ by at least one subject. To determine whether $U$ ratings clustered at the lower end of the typicality range, each category was divided into the upper and lower halves of the typicality range (as determined by the ratings in Experiment 2), and the number of $U$ ratings in each half of the range was counted for each category and then averaged across the eight categories. For the 16 subjects who gave $U$ ratings, all gave more to category members in the lower half of the typicality range. This difference was significant by a Wilcoxon matched-pairs signedranks test $(\mathrm{p}<.01)$.

It might also be noted that for three of the categories (clothing, furniture, and vehicle), there were virtually no $U$ ratings given by any subject, but for the remaining categories, as many as seven members received Us from a single subject. These three categories were all at the superordinate level, and hence the names of their members should be at the basic or most commonly used level of abstraction (Rosch, Mervis, Gray, Johnson, \& Boyes-Braem, 1976). Two of these categories, clothing and furniture, were two of the three categories in Experiment 2 for which the correlation between typicality and number of properties was not significant, which is also consistent with the relationship between typicality and familiarity being largely due to unfamiliar words.

The Experiment 2 correlations between typicality and mean number of properties were recalculated, omitting all items that received a $U$ rating from four or more subjects in Experiment 3 (one-fifth of the number who provided ratings). Table 1 gives the correlations before and after omitting the $U$ items. Although they remained generally positive, correlations did drop for all five categories that contained members with the criterial number of $U$ ratings. Two categories dropped somewhat below the level of significance. For two other categories, the correlations remained significant at the .05 level but not at the .01 level. The fifth category, fish, which was close to zero previously, became slightly negative. (Lowering the criterion for omitting exemplars from four to three $U$ ratings caused the correlation for fruit to drop to a nonsignificant .49 and also caused the correlation for fish to become more negative, -.44 .)

It should be noted that omitting the $\mathrm{U}$ exemplars from the sample reduced the range of number of properties produced but left the range of typicality essentially the same (for four of the five categories, the

Table 1

Correlation (r) Between Rated Typicality and Number of Properties Produced, Before and After Removal of Unfamiliar (U) Exemplars

\begin{tabular}{llcccc}
\hline & $\begin{array}{c}\text { Before Removal } \\
\text { (Experiment 2) }\end{array}$ & & \multicolumn{2}{c}{$\begin{array}{c}\text { After } \\
\text { Removal }\end{array}$} \\
\cline { 2 - 5 } \cline { 4 - 5 } & $\mathrm{r}$ & $\mathrm{n}$ & & $\mathrm{r}$ & $\mathrm{n}$ \\
\hline Birds & $.68 \dagger$ & 15 & $.60^{*}$ & 13 \\
Clothing & .24 & 15 & .24 & 15 \\
Fish & .07 & 15 & -.06 & 13 \\
Flowers & $.69 \dagger$ & 15 & .62 & 9 \\
Fruit & $.66 \dagger$ & 15 & $.62^{*}$ & 14 \\
Furniture & .36 & 15 & .36 & 15 \\
Tree & $.67 \dagger$ & 15 & .44 & 13 \\
Vehicle & $.62^{*}$ & 15 & $.62^{*}$ & 15 \\
\hline
\end{tabular}

${ }^{*} p<.05$ (two-tailed test). $\quad t_{p}<.01$ (two-tailed test). 
typicality range was unchanged; for the fifth, it decreased by 1.25 ). Removing $U$ exemplars simply excluded lowrated exemplars that were unknown to subjects, while retaining equally atypical exemplars whose properties were at least somewhat known. The reductions in correlation thus were not caused by truncating the range of typicality.

Thus, after omitting words for which subjects did not have minimal knowledge of the referent, only three of eight correlations remained statistically significant. The relationship between familiarity and typicality observed in Experiment 2 may therefore be partly attributed to a response strategy for dealing with unfamiliar words. All correlations except one did, however, remain substantially positive. This fact suggests that familiarity of referents may have some influence on perceived typicality, as proposed by Ashcraft (1978b).

\section{GENERAL DISCUSSION}

These experiments indicate that familiarity alone cannot account for variations in typicality, although it may indeed contribute to them. Experiment 1 showed that subjects were not necessarily less familiar with items rated low in typicality than with those high in typicality, when familiarity was measured by number of properties produced. Experiment 2 showed that in a large, random sample of items, there was a tendency for items rated low in typicality to have fewer properties listed, replicating Ashcraft (1978b), but results from Experiment 3 suggested that part of the reason for this result may be a response bias toward assigning totally unfamiliar items to the bottom of the scale, rather than a reflection of low perceived typicality of the referents themselves. These results cast doubt on Ashcraft's (1978a) suggestion that familiarity is the variable underlying typicality effects in semantic memory tasks such as verification times. It still remains to be demonstrated that standard typicality effects will be obtained for exemplars that are equal in number of properties produced. However, in Experiment 1, familiarity and typicality were negatively correlated; if mean number of properties produced is indeed a better predictor of reaction times than is rated typicality, as Ashcraft (1978b) found, then reaction time should be faster to items at the lower end of the bird sample used in Experiment 1, a prediction that contradicts a substantial body of findings (e.g., Smith et al., 1974).

In addition to the present results, another finding concerning natural categories indicates that typicality differences need not arise from variations in familiarity. Smith et al. (1974) found that the relative typicality of two exemplars could change depending on what category was being considered. For example, although a robin was rated as a more typical bird than a chicken, chickens were considered to be the more typical animal. It would seem to be true for many items that their typicality may vary depending on the category they are rated in relation to. A snake may be a typical reptile, a moderately typical vertebrate, and an atypical animal.

It therefore seems reasonable to conclude that differences in typicality can exist independent of any level of familiarity. The fact that most of the correlations in Experiment 3 remained positive after removal of totally unfamiliar items does suggest, however, that familiarity played some part in determining the typicality ratings of Experiment 2 items. Category members that are recognizable to subjects but are still lower in familiarity than others may tend to be rated lower in typicality than the familiar ones, particularly if their names are ones that are not frequently heard. Perhaps the safest conclusion is that more than one factor can influence typicality ratings: Category members that are familiar may be low in typicality if, like chickens, coconuts, and subways, they have properties uncommon within the category. Others, such as dogwood trees, ravens, and cherry blossoms, may be given low ratings if they are less familiar to subjects than many of the other category members. And some, such as catbirds, jonquils, and ginko trees, may simply be assigned low ratings despite their similarity to typical members of their categories because subjects do not know their appearance. The extent to which the various factors come into play may depend in part on the general level of familiarity of the exemplars. For categories such as clothing, in which most exemplars are quite well-known, properties of the members may be the main determinant of ratings; for others, such as trees, familiarity may be more heavily weighted.

\section{REFERENCES}

Ashcraft, M. H. Property dominance and typicality effects in property statement verification. Journal of Verbal Learning and Verbal Behavior, 1978, 17, 155-164. (a)

Ashcraft, M. H. Property norms for typical and atypical items from 17 categories: A description and discussion. Memory \& Cognition, 1978, 6, 227-232. (b)

Battig, W. F., \& Montague, W. E. Category norms for verbal items in 56 categories: A replication and extension of the Connecticut category norms. Journal of Experimental Psychology Monograph, 1969, 80(3, Pt. 2).

Glass, A. L., \& Mrany, P. J. Evidence for two kinds of lowtypical instances in a categorization task. Memory \& Cognition, $1978,6,622-628$.

Kučera, H., \& Francis, W. N. Computational analysis of present-day American English. Providence, R.I: Brown University Press, 1967.

McCloskey, M. The stimulus familiarity problem in semantic memory research. Journal of Verbal Learning and Verbal Behavior, 1980, 19, 485-502.

Mervis, C. B., Catlin, J., \& Rosch, E. Relationships among goodness-of-example, category norms, and word frequency. Bulletin of the Psychonomic Society, 1976, 7, 283-284.

Mervis, C. B., \& Rosch, E. Categorization of natural objects. Annual Review of Psychology, 1981, 32, 89-115.

Rips, L. J., Shoben, E. J., \& Smith, E. E. Semantic distance and the verification of semantic relations. Journal of Verbal Learning and Verbal Behavior, 1973, 12, 1-20. 
Rosch, E. Cognitive representations of semantic categories. Journal of Experimental Psychology: General, 1975, 104, 192233.

Rosch, E., \& Mervis, C. B. Family resemblances: Studies in the internal structure of categories. Cognitive Psychology, 1975, 7, 573-605.

Rosch, E., Mervis, C. B., Gray, W. D., Johnson, D. M., \& Boyes-Braem, P. Basic objects in natural categories. Cognitive Psychology, 1976, 8, 382-439.

Rosch, E., Simpson, C., \& Miller, S. Structural bases of typicality effects. Journal of Experimental Psychology: Human Perception and Performance, 1976, 2, 491-502.

Smith, E. E., Shoben, E. J., \& Rips, L. J. Structure and process in semantic memory: A featural model for semantic decisions. Psychological Review, 1974, 81, 214-241.

\section{NOTES}

1. There is a distinction to be made between category members such as robins, which are familiar because they are probably frequently encountered in the environment, and those such as penguins, for which knowledge of the many properties listed is more likely to be acquired from stories and pictures. The property-listing method does not distinguish between these two kinds of familiarity. It is doubtful that our results in any way hinge on this distinction, however, since all the furniture members, atypical as well as typical, are probably equally likely to be encountered in the environment.

2. We favored Rosch, Mervis, Gray, Johnson, and BoyesBraem's (1976) biological basic-level categories because they tended to have more members with relatively simple names.

3. Another possible explanation of the different outcomes in Experiments 1 and 2 is that the perceived typicality of an exemplar or the number of properties generated for it may depend on the general context in which it is presented. The difference in context, not the difference in exemplars per se, might then be the source of the difference in results between the two experiments. A means of evaluating this possibility is to see whether the results for those bird and furniture exemplars that appeared in both experiments were substantially different. There were five such birds and seven such furniture items. Correlations between the typicality ratings given to these items in Experiments 1 and 2 were extremely high (.92 for the birds and .98 for the furniture); thus, typicality ratings were not influenced by the different item sets. Agreement for the mean number of properties produced between the two experiments was moderate for birds $(\mathrm{r}=.47)$, but negligible for furniture $(\mathrm{r}=.15)$. However, the range of properties for this particular sample of seven furniture items was smaller than the average standard deviation around any one point, suggesting that no correlation could be expected. Overall, then, there does not seem to be strong evidence to support a context-dependency explanation of differing results for the two experiments.

Appendix A

Exemplars, Mean Typicality Ratings (TR), and Mean Number of Properties Produced (NP) for Experiment 1

\begin{tabular}{lcclccc}
\hline & \multicolumn{3}{c}{ Birds } & & & \multicolumn{3}{c}{ Furniture } \\
\cline { 1 - 2 } \cline { 5 - 6 } Exemplar & TR & NP & & Exemplar & TR & NP \\
robin & 6.89 & 8.00 & & sofa & 6.79 & 7.80 \\
bluebird & 6.42 & 6.90 & chair & 6.74 & 6.85 \\
seagull & 6.26 & 7.25 & table & 6.74 & 6.80 \\
swallow & 6.16 & 5.95 & desk & 6.42 & 7.90 \\
falcon & 5.75 & 7.85 & dresser & 6.21 & 6.40 \\
mockingbird & 5.47 & 7.00 & bed & 6.16 & 8.85 \\
starling & 5.16 & 7.35 & bookcase & 5.37 & 6.50 \\
owl & 5.00 & 8.65 & piano & 4.82 & 8.50 \\
vulture & 4.84 & 8.65 & footstool & 4.74 & 6.60 \\
sandpiper & 4.47 & 6.85 & lamp & 4.52 & 7.70 \\
chicken & 3.95 & 9.15 & mirror & 3.47 & 7.20 \\
flamingo & 3.37 & 8.60 & cushion & 3.26 & 7.15 \\
albatross & 3.32 & 7.65 & vase & 2.79 & 7.25 \\
penguin & 2.63 & 9.60 & clock & 2.63 & 9.15 \\
bat & 1.53 & 8.85 & rug & 2.63 & 7.15 \\
& & & picture & 2.58 & 7.10 \\
& & & radio & 2.53 & 8.95 \\
& & & stove & 2.53 & 8.60 \\
& & & closet & 2.00 & 6.20 \\
& & & telephone & 1.74 & 8.75 \\
\hline
\end{tabular}

Appendix B

Exemplars, Mean Typicality Ratings (TR), and Mean Number of Properties Produced (NP) for Experiment 2

\begin{tabular}{|c|c|c|c|c|c|c|c|c|c|c|c|}
\hline \multicolumn{3}{|c|}{ Birds } & \multicolumn{3}{|c|}{ Clothing } & \multicolumn{3}{|c|}{ Fish } & \multicolumn{3}{|c|}{ Flowers } \\
\hline Exemplar & TR & NP & Exemplar & TR & $\mathrm{NP}$ & Exemplar & TR & NP & Exemplar & TR & NP \\
\hline sparrow & 6.30 & 8.13 & shirt & 7.00 & 9.00 & trout & 6.65 & 8.06 & rose & 6.95 & 8.06 \\
\hline robin & 6.25 & 9.75 & dress & 6.55 & 6.63 & salmon & 6.30 & 8.88 & daisy & 6.60 & 8.69 \\
\hline bluejay & 5.60 & 8.69 & slacks & 6.45 & 7.19 & tuna & 6.20 & 7.69 & poppy & 5.20 & 7.38 \\
\hline crow & 5.30 & 8.63 & coat & 5.90 & 7.69 & goldfish & 6.15 & 7.50 & lily & 4.95 & 7.44 \\
\hline hawk & 5.15 & 8.50 & socks & 5.50 & 7.94 & minnow & 5.05 & 7.88 & iris & 4.75 & 6.44 \\
\hline wren & 4.50 & 7.56 & underpants & 5.40 & 7.75 & carp & 4.70 & 6.94 & marigold & 4.45 & 8.31 \\
\hline duck & 4.35 & 9.75 & belt & 4.95 & 6.50 & sardine & 4.65 & 8.56 & African violet & 4.35 & 5.63 \\
\hline owl & 4.25 & 9.00 & sweatshirt & 4.55 & 7.44 & shark & 3.75 & 9.06 & lilac & 4.10 & 7.31 \\
\hline mockingbird & 4.10 & 6.75 & bathrobe & 4.15 & 6.31 & whitefish & 3.45 & 6.25 & azalea & 3.90 & 6.50 \\
\hline chicken & 4.05 & 7.81 & scarf & 3.30 & 7.75 & sunfish & 3.15 & 5.19 & cherry blossom & 3.80 & 6.63 \\
\hline raven & 3.95 & 7.81 & gloves & 2.55 & 7.94 & shrimp & 2.70 & 8.69 & peony & 3.45 & 4.69 \\
\hline thrush & 3.50 & 7.25 & watch & 2.50 & 8.31 & dolphin & 2.70 & 8.50 & begonia & 3.35 & 7.25 \\
\hline pelican & 3.20 & 8.25 & necklace & 2.35 & 7.88 & lobster & 2.40 & 8.63 & jonquil & 3.05 & 4.69 \\
\hline catbird & 2.55 & 3.38 & cape & 2.05 & 6.19 & walleye fish & 2.35 & 6.75 & sweet pea & 2.75 & 6.94 \\
\hline albatross & 2.30 & 6.19 & cane & 1.65 & 6.19 & eel & 1.85 & 8.50 & dogwood blossom & 2.55 & 5.13 \\
\hline
\end{tabular}


Fruit

\begin{tabular}{lrr}
\hline Exemplar & TR & \multicolumn{1}{c}{ NP } \\
\hline apple & 6.90 & 11.63 \\
peach & 6.30 & 9.13 \\
strawberry & 6.25 & 9.13 \\
pear & 6.20 & 7.88 \\
grape & 5.60 & 10.19 \\
blueberry & 4.90 & 8.88 \\
lemon & 4.85 & 9.88 \\
watermelon & 4.75 & 9.88 \\
raisin & 3.55 & 8.31 \\
fig & 3.05 & 7.75 \\
coconut & 2.60 & 9.13 \\
pumpkin & 2.45 & 8.81 \\
pawpaw & 1.55 & 3.56 \\
olive & 1.50 & 8.50 \\
gourd & 1.50 & 5.63 \\
\hline
\end{tabular}

Furniture

\begin{tabular}{lcc}
\hline \multicolumn{1}{c}{ Exemplar } & TR & NP \\
\hline chair & 6.85 & 6.63 \\
table & 6.65 & 7.25 \\
sofa & 6.65 & 7.31 \\
bureau & 5.10 & 7.25 \\
lounge chair & 5.00 & 6.75 \\
television & 4.70 & 9.38 \\
bench & 4.55 & 7.06 \\
shelf & 3.80 & 6.56 \\
rug & 3.65 & 7.25 \\
mirror & 3.60 & 6.25 \\
cushion & 3.35 & 6.88 \\
chaise lounge & 3.25 & 5.75 \\
cedar chest & 3.15 & 6.75 \\
drapes & 3.15 & 6.44 \\
vase & 2.55 & 6.25 \\
\hline
\end{tabular}

\begin{tabular}{lcc}
\multicolumn{3}{c}{ Trees } \\
\hline Exemplar & TR & NP \\
pine & 6.55 & 9.31 \\
oak & 6.45 & 7.75 \\
maple & 6.25 & 6.56 \\
redwood & 5.80 & 7.88 \\
elm & 5.70 & 6.94 \\
sequoia & 4.75 & 8.19 \\
palm & 4.60 & 6.56 \\
beech & 4.45 & 5.19 \\
peach & 4.30 & 7.25 \\
pear & 4.25 & 6.75 \\
cypress & 4.20 & 6.31 \\
dogwood & 3.80 & 5.31 \\
mimosa & 2.85 & 2.38 \\
ginko & 2.60 & 1.56 \\
bamboo & 2.45 & 7.44
\end{tabular}

\begin{tabular}{lcr}
\multicolumn{3}{c}{ Vehicles } \\
\hline Exemplar & TR & NP \\
\hline car & 6.25 & 9.00 \\
truck & 6.20 & 8.00 \\
airplane & 5.75 & 10.38 \\
bicycle & 5.75 & 8.69 \\
train & 5.60 & 8.75 \\
van & 5.40 & 8.94 \\
jeep & 4.70 & 7.56 \\
subway & 3.65 & 9.50 \\
cable car & 3.45 & 8.31 \\
feet & 3.00 & 7.31 \\
rowboat & 3.00 & 7.06 \\
horse & 2.80 & 9.19 \\
raft & 2.45 & 7.19 \\
go-cart & 2.35 & 7.25 \\
dogsled & 1.90 & 6.25 \\
\hline
\end{tabular}

(Received for publication April 15, 1981; revision accepted July $16,1981$. 